\title{
Chaotic resonances of a Bose-Einstein condensate in a cavity pumped by a modulated optical field
}

\author{
M. Diver ${ }^{*}$ G.R.M. Robb, and G.-L. Oppo \\ Institute of Complex Systems, SUPA, and Department of Physics, \\ University of Strathclyde, Glasgow, G4 ONG, Scotland
}

(Dated: January 26, 2015)

\begin{abstract}
We present a theoretical analysis of a Bose-Einstein condensate (BEC) enclosed in an optical cavity driven by a modulated external laser beam where the cavity-field variable is adiabatically eliminated. The modulation of the amplitude of the pump laser induces nonlinear resonances and the widespread presence of chaotic oscillations even when repulsive atom-atom interactions are negligible. Close to resonance, varying the modulation amplitude by just a few percent causes abrupt and erratic changes to the output laser intensity with peak power increasing by almost an order of magnitude. We also use a simplified model of the BEC-cavity system that considers only a small number of spatial modes of the BEC to show that, despite the disruptive presence of a modulation in the pump beam, the system can still be considered to be low-dimensional.
\end{abstract}

PACS numbers:

Keywords:

\section{INTRODUCTION}

The introduction of chaos can, when coupled with a stabilising mechanism, increase the utility of a device by allowing the selection of an appropriate system state that would otherwise be difficult to achieve [1]. Accordingly, methods that can aid the transition of a system to a chaotic state can be of great benefit. One such method is the application of a parameter modulation, which can, by the selection of an appropriate frequency, lower the threshold of the onset of chaos [2, 3].

Interest in the study of Bose-Einstein condensates (BECs) confined to optical cavities has continued to develop in recent years, both theoretically 449 and experimentally [10-14. The coupling between the light field of the optical cavity and the wave function of the BEC in such a system can result in chaotic dynamics. Such dynamics in BECs have been investigated for a variety of theoretical and experimental setups (see, e.g., [15 18]). It has been previously shown that chaotic dynamics arising from the nonlinear atom-field interaction are an intrinsic feature of the BEC-cavity system; chaos occurs sporadically in the domain where interactions between condensate atoms are negligible, and ubiquitously when atom-atom interactions are included [19. In both cases, the chaotic oscillations appear beyond a critical intensity of the input pump laser.

In this paper, we investigate the resonance effect of a pump amplitude modulation on the dynamics of the cavity-confined condensate. The effect of modulating the pump-beam power in a simplified model of a BEC-cavity system was previously considered in [20, where it was shown that the introduction of this modulation could be applied as an optical Kerr switch. In 20, the dynamics of

*Corresponding author: (martin.diver@strath.ac.uk) the BEC are approximated both by considering only two spatial modes of the condensate and by neglecting the interactions between atoms, a setup previously described in 21. Here, we consider the effect of a modulation using first the full model, which imposes no restriction on the number of spatial modes of the BEC, and then a simplified model involving only a few (but more than two) spatial modes of the BEC. It is shown that the addition of a pump amplitude modulation is roughly analogous to the effect of atom-atom interactions in the BEC-cavity system [19]: in the absence of pump modulation, the system exhibits sporadic instances of chaos, whereas in the presence of pump amplitude modulation and close to resonance, chaotic oscillations can be found extensively through a wide parameter range. The results presented suggest that use of a modulated pump beam could be a simple and effective method for inducing chaotic dynamics for a wide range of system parameters. In addition, inducing chaos via pump modulation should be easier to realise in practice than inducing chaos via manipulation of the atom-atom interaction.

We first consider the effects that pump intensity and strength of atom-atom interactions have on the fundamental temporal frequency of the BEC-cavity system's oscillations without external modulations. We then proceed to find resonance frequencies and the associated modulation amplitudes required to induce chaotic dynamics in the presence of a modulated pump. We illustrate our findings by displaying the evolution in phase space of the BEC-cavity system, along with the associated phase-space orbit "widths" that provide a useful indicator of the presence of chaos, Poincaré sections depicting the presence of both quasiperiodicity and chaos, the cavity intensity as it responds to the backaction of the BEC oscillations, and representative power spectra. We also present spatial spectra for the system that indicate the small number of modes (typically five) required for an accurate description of the BEC-cavity system's 


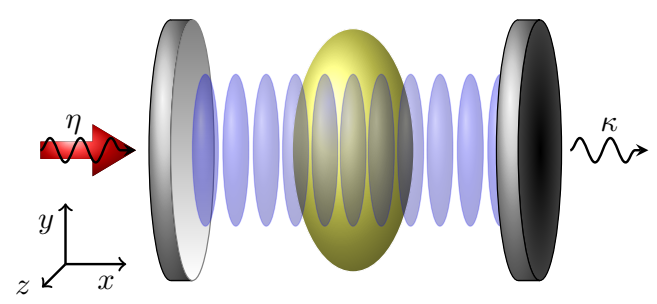

FIG. 1: (Color online.) The BEC-cavity system, where the single-mode cavity field interacts with a cigar-shaped condensate that is aligned transversely to the cavity axis. The cavity is driven by a pump laser represented by the pump rate $\eta$ with a modulation of frequency $\Omega_{m}$ and relative amplitude $\mu$; cavity losses are represented by the decay rate $\kappa$.

dynamics. As a consequence, we introduce a simplified $n$-mode model to describe the BEC-cavity system's behaviour, and, for our range of parameters, show excellent agreement for the chaotic resonances between the full model and simplified $n$-mode model.

\section{THE BEC-CAVITY SYSTEM}

We consider a ${ }^{87} \mathrm{Rb}$ BEC composed of $N=10^{5}$ atoms, each with mass $m=1.44 \times 10^{-25} \mathrm{~kg}$, confined to a FabryPérot optical cavity driven by an external blue-detuned laser beam of wavelength $\lambda=780 \mathrm{~nm}$, with a cavity loss rate of $\kappa=2 \pi \times 1.3 \mathrm{MHz}$, as shown schematically in Fig. 1. The system setup is as described in [19]; the BEC couples to a single mode of the cavity, where the detuning between the pump frequency $\omega_{p}$ and that of the cavity $\omega_{c}$ is given by $\Delta_{c}=\omega_{p}-\omega_{c}$. Effects arising from spontaneous emission are disregarded by assuming a large detuning $\Delta_{a}$ between the pump and the resonant frequency of the atoms $\omega_{a}$, where $\Delta_{a}=\omega_{p}-\omega_{a}=2 \pi \times 58 \mathrm{GHz}$. This latter detuning determines the depth of the standingwave cavity mode, given by $\hbar U_{0}$, where $U_{0}=g_{0}^{2} / \Delta_{a}$, with $g_{0}=2 \pi \times 14.1 \mathrm{MHz}$ being the maximum atomphoton coupling strength. Note that the chosen parameters have previously been used in both theoretical and experimental investigations [19, 22. It should be emphasised, however, that the results are representative of a wide range of parameter values.

The BEC-cavity system is pumped externally by an optical beam with an average amplitude corresponding to a mean pump rate $\eta$. This beam is then modulated with an amplitude relative to the mean pump amplitude denoted by $\mu$ and at a frequency of $\Omega_{m}$.

Since the optical lattice is aligned with the cavity axis [22], we use a one-dimensional model of the condensate dynamics, which can be derived from the threedimensional Gross-Pitaevskii equation as described in, e.g., 23 26]. The BEC-cavity system is described by the following coupled equations:

$$
\begin{aligned}
\frac{\partial \psi(x, t)}{\partial t} & =\frac{i}{2} \frac{\partial^{2} \psi}{\partial x^{2}}-i|\alpha|^{2} V_{0} \sin ^{2}\left(\frac{\pi x}{2}\right) \psi-i \Lambda|\psi|^{2} \psi, \\
|\alpha|^{2} & =\frac{\eta^{2}\left[1+\mu \cos \left(\omega_{m} t\right)\right]}{\kappa^{2}+\left(\Delta_{c}-U_{0} N\left\langle\psi\left|\sin ^{2}(\pi x / 2)\right| \psi\right\rangle\right)^{2}},
\end{aligned}
$$

where $x$ is a dimensionless position variable scaled with respect to half the lattice period $L$, where $L=\lambda / 2$. Similarly, $t$ is a dimensionless time variable scaled with respect to $T_{0}=m L^{2} / 4 \hbar$, and $\Lambda=2 g_{1 \mathrm{D}} N T_{0} /(\hbar L)$, $V_{0}=U_{0} T_{0}, \omega_{m}=\Omega_{m} T_{0}$ are dimensionless parameters that characterise the scattering length, BEC-lattice coupling strength, and modulation frequency respectively. In the definition of $\Lambda$, the atom-atom interaction parameter $g_{1 \mathrm{D}}=2 \hbar \omega_{\perp} a$, where $a$ is the scattering length and the transverse trap frequency $\omega_{\perp}$ is $\sqrt{\omega_{y} \omega_{z}}$, with the $(y, z)$ trap frequencies $\omega_{y}=2 \pi \times 37 \mathrm{~Hz}$ and $\omega_{z}=2 \pi \times 210$ $\mathrm{Hz}$. The intracavity photon number is given by $|\alpha|^{2}$, and the integral in the denominator of Eq. (2) describes the overlap of the cavity mode with the density profile of the condensate. In the examples that follow, we write the dimensionless modulation frequency $\omega_{m}$ in terms of a dimensionless recoil frequency $\omega_{r}=4 \Omega_{r} T_{0}$, with the recoil frequency $\Omega_{r}=\left(\hbar k^{2}\right) /(2 m)$ [5], and $k=2 \pi / \lambda$, so that $\omega_{r}=\pi^{2} / 2$.

The dimensionless BEC wave function $\psi$ is normalised such that $\langle\psi \mid \psi\rangle=1$. In this model, the cavity-field dynamics are adiabatically eliminated since we consider the limit where the decay rate $\kappa>>\Omega_{r}$.

We initially focus in Section 4 on a modulated-pump system where the coupling between atoms is solely through the cavity field and atom-atom interactions are neglected (i.e., $\Lambda=0$ ). In Section 5 we also include the effect of atom-atom interactions in a repulsive condensate.

Given the previously established low-dimensional nature of the system without modulation [19, it is reasonable to investigate whether the modulated system retains this curious feature. In Section 6 we accordingly apply a truncated model that reduces the wave function as given by Eq. (1) to a system composed of only five motional states. We show that for the relevant areas of interest, a close correspondence between the results from the two models persists and that the dynamical behaviour of the system remains low-dimensional, despite the introduction of pump modulation.

\section{FREQUENCY RESPONSE IN THE ABSENCE OF MODULATION}

It can be seen from Eq. (1) that in the absence of cavity-field or atom-atom interactions, the BEC will evolve freely, with each spatial mode oscillating at a rate proportional to $\omega_{r}=\pi^{2} / 2$. This frequency arises from 
the term of Eq. (1) that governs the kinetic energy of the BEC. The presence of light-matter (parameter $\eta / \kappa$ ) and atom-atom (parameter $\Lambda$ ) interactions, however, introduce important nonlinear shifts in the response of the BEC-cavity system without modulations.

In Fig. 2 we show with black dashed lines the changes of the fundamental frequency for a range of $\eta / \kappa$ values, for the case where no atom-atom interactions are present $(\Lambda=0)(\mathrm{a})$, along with the dominant frequencies from two particular values of $\eta / \kappa(b)$. Both plots are generated from numerical simulations of the frequency spectra of Eqs. (1) and (2) with no pump modulation $(\mu=0)$ using the following procedure: the temporal evolution of the cavity field is calculated numerically using Eqs. (1) and (2), and is then converted to frequency spectra using a fast Fourier transform. The resulting frequency spectrum of the cavity field is then calculated for a range of values of $\eta / \kappa$, and the dominant frequency from each spectrum is recorded. These dominant frequencies are then plotted as the black dashed line in (a).

We extend the analysis presented in Section V B of [19] to obtain a simple expression for the frequency of the unmodulated system. We decompose the BEC wave function $\psi(x, t)$ into a series of spatial modes with timedependent amplitudes

$$
c_{n}(t)=\frac{1}{L_{D}} \int_{-L_{D} / 2}^{L_{D} / 2} \psi(x, t) \mathrm{e}^{-\mathrm{i} \pi n x} d x
$$

where $L_{D}$ is the length of the BEC in units of $x$, i.e., $L_{D}=2 L_{B E C} / L$, where $L_{B E C}$ is the length of the $\mathrm{BEC}$. We then introduce the variables $R=\operatorname{Re}\left(c_{0} c_{1}^{*}\right)$, $I=\operatorname{Im}\left(c_{0} c_{1}^{*}\right)$ (similar to [21]), and $D=\left|c_{0}\right|^{2}-2\left|c_{1}\right|^{2}$ and obtain:

$$
\begin{aligned}
\frac{d R}{d t} & =-\omega_{r} I \\
\frac{d I}{d t} & =\left(\omega_{r}+\frac{\Lambda}{n_{L}}\right) R-|\alpha|^{2} \frac{V_{0}}{4} D, \\
\frac{d D}{d t} & =2 I\left(|\alpha|^{2} V_{0}-8 R \frac{\Lambda}{n_{L}}\right),
\end{aligned}
$$

where $n_{L}$ is the number of lattice sites occupied by the BEC, and $|\alpha|^{2}$ is given by Eq. (2), with the overlap integral $\left\langle\psi\left|\sin ^{2}(\pi x / 2)\right| \psi\right\rangle$ approximated to $1 / 2-2 R$. The frequency of the unmodulated system can then be approximated as:

$$
\omega=\sqrt{\omega_{r}} \sqrt{\omega_{r}-F\left(\frac{\eta}{\kappa}\right)+\frac{\Lambda}{n_{L}}}
$$

where $F$ is a polynomial function of $\eta / \kappa$. The red dotdashed curve in Fig. 2 a shows the frequency of the unmodulated system (7) where $F$ is a quartic polynomial in $\eta / \kappa$. The level of agreement with the results of the numerical simulations is sufficiently high that the numerical (black dashed) and analytical (red dot-dashed) lines have been displaced so as to be discernible in the plot. A similar level of agreement has been obtained for cases where atom-atom interactions are also present $(\Lambda>0)$. When a small modulation is introduced (see the solid blue line in Fig. 2 a for the case $\mu=0.017$ ), the modulation frequency (in this case, $0.81 \omega_{r}$ ) dominates the spectrum until the onset of bistability where both the modulation and system's response are of approximately equal magnitude (transition region). Fig. $2 \mathrm{~b}$ shows the dominant oscillation frequencies for $\eta / \kappa=0.37$ and $\eta / \kappa=0.41$. Note that both peaks for each value of $\eta / \kappa$, are of a similar magnitude; small fluctuations between these values account for the "jumps" between the modulation frequency and system frequency given in Fig. 2 for the $\eta / \kappa$ in the range 0.37-0.41. Above the transition region the external and system's response resonate and induce highly nonlinear oscillations. For example, the blue cross in Fig. 2a at $\eta / \kappa=0.90$ indicates the presence of chaos induced by the modulation.

The knowledge of the dynamical behaviour of the unmodulated system is then important for the determination of the resonant frequencies when a pump modulation is introduced. For this reason, we show in Fig. 3 a strong agreement between the results of the full numerical simulation of Eqs. (1) and (2) with $\mu=0$ and the approximate expression (7) when modifying the atomatom interaction coefficient $\Lambda$. Here, the pump rate is first set to $\eta / \kappa=0.01$ where the pump's influence can be neglected, and then to $\eta / \kappa=0.75$ where the system's dynamics with modulations is dominated by its natural response.

\section{PUMP MODULATION OF AN INTERACTION-FREE CONDENSATE}

We first consider the case where collisions between condensate atoms are neglected, i.e., $\Lambda=0$. We investigate the system numerically to determine the lowest value of the pump modulation amplitude, $\mu$, at which chaotic oscillations appear. To ensure that chaotic behaviour is being induced by the modulation of the pump amplitude, we investigate cases where only periodic or quasiperiodic oscillations occur in the unmodulated case, i.e., where the value of $\eta$ is comfortably below the critical pump rate $\eta_{\text {cr }}=1.07$ [19]. We proceed by setting $\eta / \kappa=0.90$, which meets the condition of $\eta / \kappa<\eta_{\mathrm{cr}}$ required to ensure (quasi-) periodic dynamics.

We have then performed a scan of pump modulation frequency to reveal the system's fundamental and harmonic resonances, as demonstrated in Fig. 4. which shows the value of the modulation amplitude $\mu$ necessary to cross the separatrix as a function of the modulation frequency $\omega_{m}$. A clear resonance appears at $\omega_{m}=0.81 \omega_{r}$, the frequency of the unmodulated system as shown in Fig. 2 for $\eta / \kappa=0.90$. Here, an extremely small value of the modulation amplitude, $\mu=0.017$ is required to induce chaotic dynamics. It is interesting to note that such a value is almost a hundred times smaller than that required to induce chaotic dynamics when off resonance 

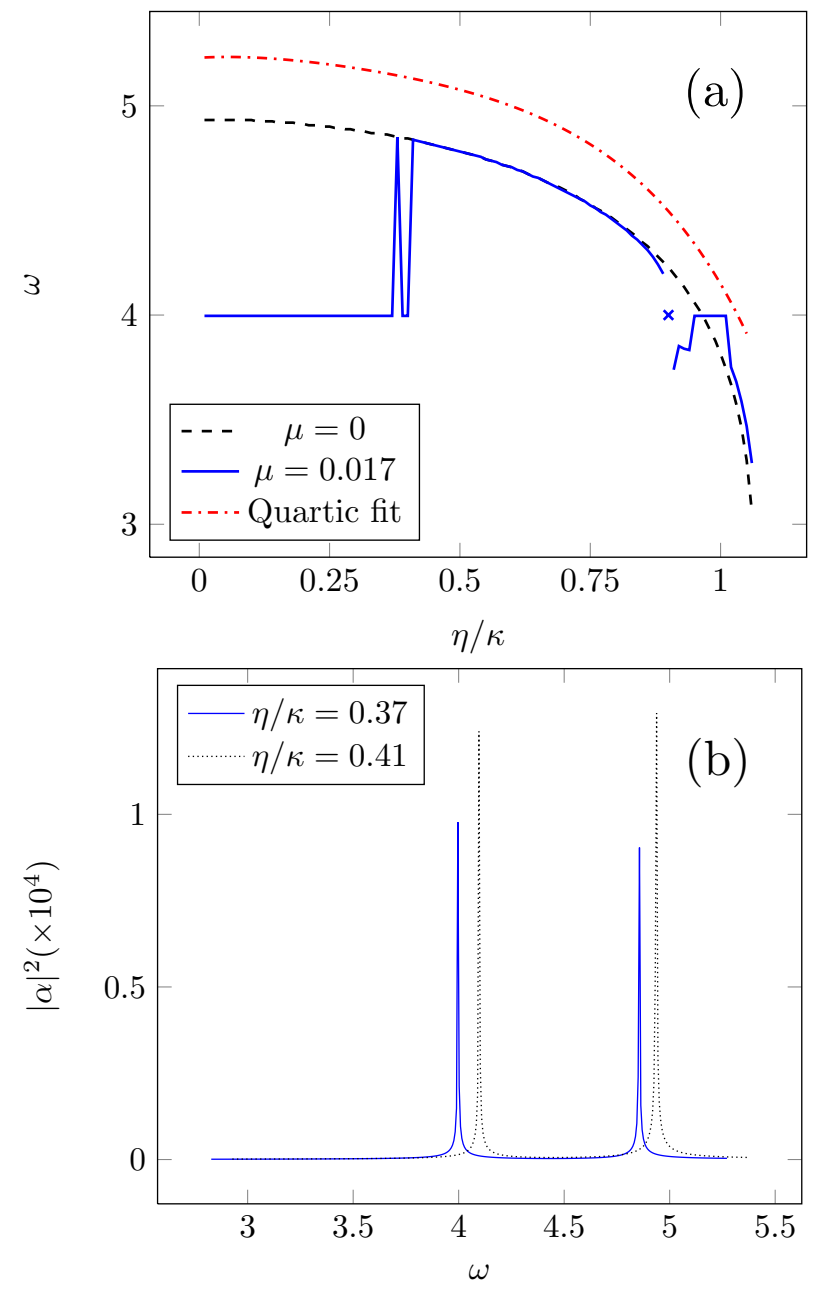

FIG. 2: (Color online.) (a) Effect of the pump intensity on the oscillation frequency of the BEC-cavity system, for the case without atom-atom interaction $(\Lambda=0)$. The black-dashed and blue-solid curves correspond to dominant frequency component from the numerical integration of Eqs. (1) and (2) for $\mu=0$ and $\mu=0.017$, respectively. The red dot-dashed line is the analytical solution Eq. (7) with a quartic fit to the function $F$; note that this curve is offset by +0.3 on the vertical axis for clarity. (b) Cavity field frequency spectra.

(e.g., when $\omega_{m}=1.1 \omega_{r}$ ). Two further resonances are identifiable in Fig. 44 one at $\omega_{m}=1.67 \omega_{r}$ and one at $\omega_{m}=0.38 \omega_{r}$, very close to the first harmonic and the first subharmonic of the unmodulated system's response studied in Section 3. As chaotic motion occurs above the curve of Fig. 4, we refer to its minima as chaotic resonances.

When a pump modulation is applied at the resonant frequency, $\omega_{m}=0.81 \omega_{r}$, the system displays drastic changes in the response of the system when the amplitude of the modulation, $\mu$, is increased. This change is illustrated in Figs. 5 and 6 , where the modulation amplitudes $\mu=0.016$ and $\mu=0.017$ are considered. The effect of the slightly larger modulation on the light field in the cavity, shown in Fig. 5, is dramatic. The initially simi-

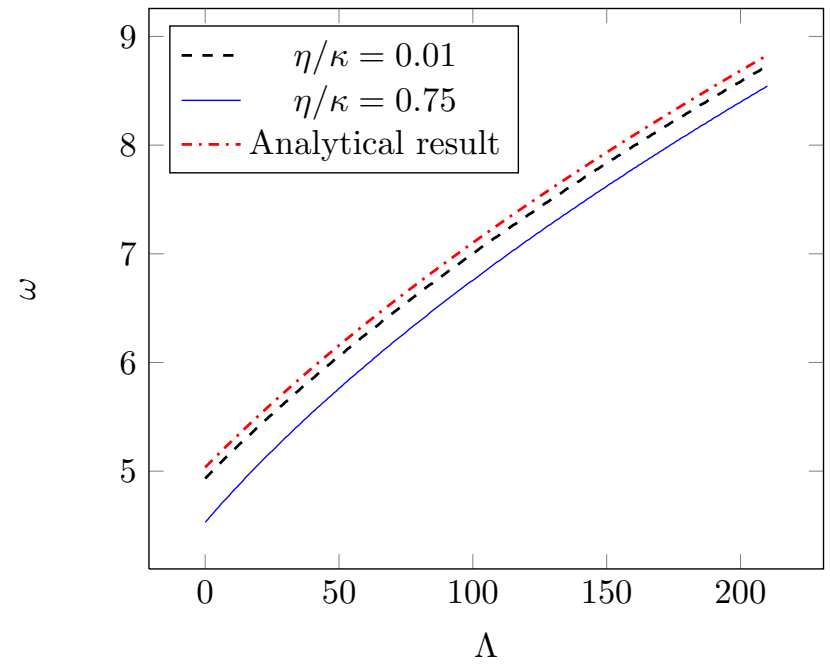

FIG. 3: (Color online.) Oscillation frequency of the unmodulated system $(\mu=0)$ for a range of values of the atom-atom interaction parameter $\Lambda$ calculated from a full numerical simulation of Eqs. (1) and (2) (dashed black line) and the expression (7) (red dot-dashed line, offset by +0.1 on the vertical axis). The dashed curve corresponds to $\eta / \kappa=0.01$; the solid (blue) curve corresponds to $\eta / \kappa=0.75$.

lar evolutions of the intracavity intensity for both values of $\mu$ diverge, slowly at first, then drastically. This can be explained by the backaction of the BEC oscillations on the cavity field; at a critical value of the modulation, the energy of the system that was previously stored in the fundamental homogeneous mode is now delivered into the cavity field. In Fig. 6, the broadband spectrum apparent for $\mu=0.017$ indicates the chaotic evolution of the light field, which contrasts with the solitary peaks apparent for $\mu=0.016$ that correspond to quasiperiodic oscillations of the BEC. Although the period of the oscillations decreases during the chaotic evolution, the adiabatic following of the field variable $\alpha$ to the atomic wave function $\psi$ remains valid.

The drastic change in dynamical response to the external modulation displayed in Figs. 5 and 6 is explained in terms of crossing a separatrix in the phase space. To illustrate this dynamical feature, we decompose the BEC wave function into a series of spatial modes of amplitude $c_{n}(t)$ given in Eq. (3). A projection of the phase space on the conjugate pair of modal variables $X=-L_{D} \sqrt{8 N} \operatorname{Re}\left(c_{0}\left(c_{1}^{*}+c_{-1}^{*}\right) / 2\right)$ and $P=$ $L_{D} \sqrt{8 N} \operatorname{Im}\left(c_{0}\left(c_{1}^{*}+c_{-1}^{*}\right) / 2\right)$ for the two modulation amplitudes $\mu=0.016$ and $\mu=0.017$ is presented in Fig. 7 (a) and (b), respectively.

In Fig. $7 \mathrm{p}$, it can be seen that the critical increase of the modulation amplitude pushes the dynamical evolution across the separatrix into a new region of high momentum $P$. Energy now is fed back in to the cavity field resulting in high-amplitude oscillations eight to ten times larger than those seen below the threshold of crossing the separatrix. Such a crossing can be used to separate the 


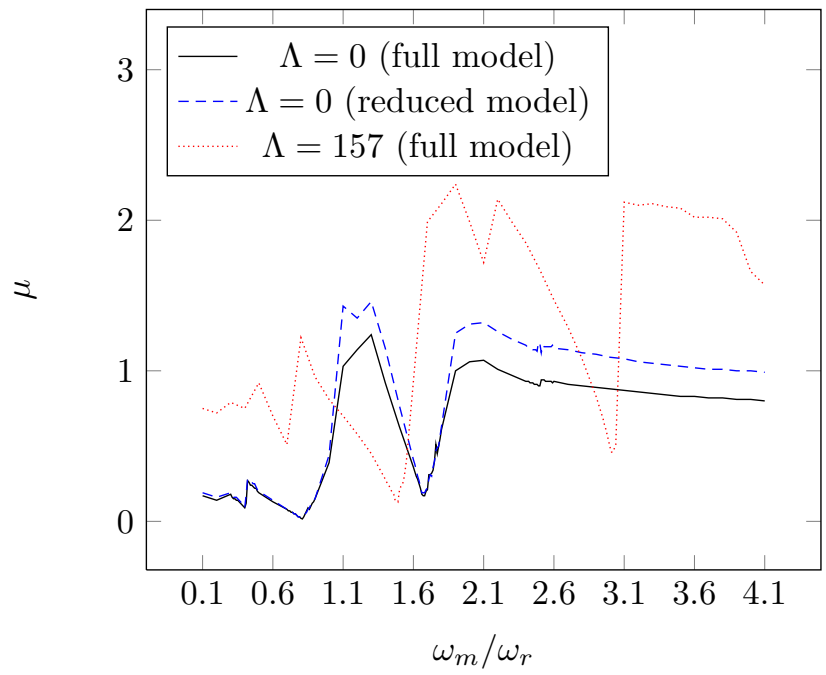

FIG. 4: (Color online.) The threshold values of modulation amplitude $\mu$ as calculated from the full model (solid black line) and the five-mode model (dashed blue line), across the modulation-frequency range of $0.1 \omega_{r}-4.1 \omega_{r}$, for $\eta / \kappa=0.90$. Two main resonances are located at $\omega_{m}=0.81 \omega_{r}$ and $\omega_{m}=$ $1.67 \omega_{r}$. The threshold value for $0.81 \omega_{r}$ is particularly small: $\mu=0.017$. The dotted red line shows the resonances when atom-atom collisions are included $(\Lambda=157)$; note the shift in the two main resonances due to the introduction atom-atom interactions $\Lambda>0$.

different dynamical regimes and in the identification of dynamical resonances (see Fig. 4). Fig. 7 also shows Poincaré sections demonstrating that the extent of the representative orbit in phase space is greatly increased once the system is in the chaotic regime across the separatrix.

Setting the modulation parameters at resonance, i.e. $\omega_{m}=0.81 \omega_{r}$ and $\mu=0.017$, the mean pump amplitude or rate $\eta$ was varied and the modulated system's behaviour was compared over a wide range of pump rates with that of the unmodulated system (see Fig. 8). Over the entire extent of our parameter range, it can be seen that the modulated case displays almost exclusively chaotic behaviour, even at pump rates below the critical pump rate $\eta_{\mathrm{cr}} \approx 1.07 \kappa$ required to (sporadically) induce chaotic motion in the interaction-free BEC-cavity system [19. This effect is similar to that achieved by taking into account the nonlinear collisional interaction between the atoms of the condensate in the un-modulated case, although a consequence of the atom-atom interactions is that $\eta_{\text {cr }}$ is increased.

\section{PUMP MODULATION OF A REPULSIVE CONDENSATE}

We extend our model by introducing two-body collisions in the BEC and considering $\Lambda>0$, i.e., a BEC with repulsive atom-atom interactions. It has previously

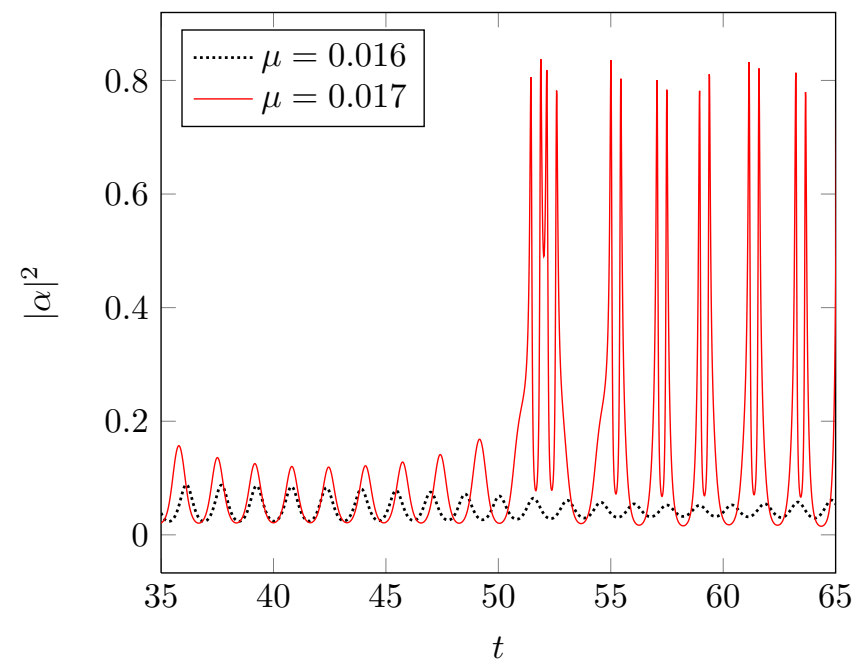

FIG. 5: (Color online.) The temporal evolution of the intracavity intensity, for $\eta / \kappa=0.90$ and $\omega_{m}=0.81 \omega_{r}$, showing, for $\mu=0.016$, quasiperiodic oscillations over the duration of the simulation, and, for $\mu=0.017$, the crossing of the separatrix (at $t=51$ ). The substantial increase in magnitude of the intracavity intensity demonstrates the sporadic transfer of energy into the cavity field.

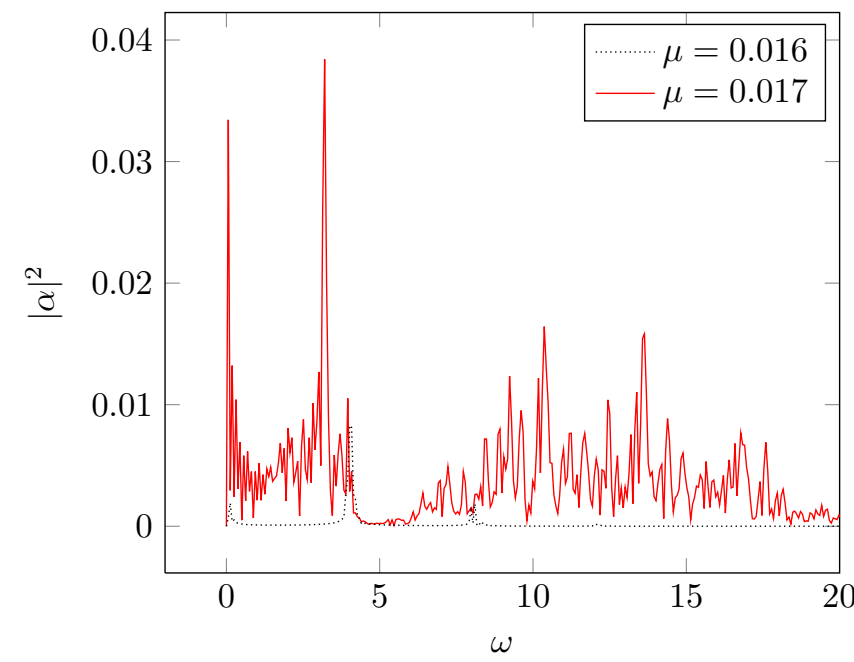

FIG. 6: (Color online.) Frequency spectra for $\mu=0.016$ (black dots) and $\mu=0.017$ (red line). The lower value of $\mu$ exhibits an output suggestive of periodic dynamics (though here there is an additional, incommensurate, frequency not apparent in the spectrum, leading to quasiperiodic dynamics). The larger value of $\mu$, however, gives a broadband spectrum that indicates the presence of chaos.

been shown that with $\Lambda>0$ and in the absence of pump modulation, chaotic dynamics are ubiquitous when the pump rate exceeds a critical value of the pump intensity $\eta_{\text {cr }}$ 19. Similarly, in section 4 we have shown that chaotic dynamics are ubiquitous in the collisionless, or interaction-free, case $(\Lambda=0)$ when the pump modulation amplitude exceeds a critical value. In this section, 

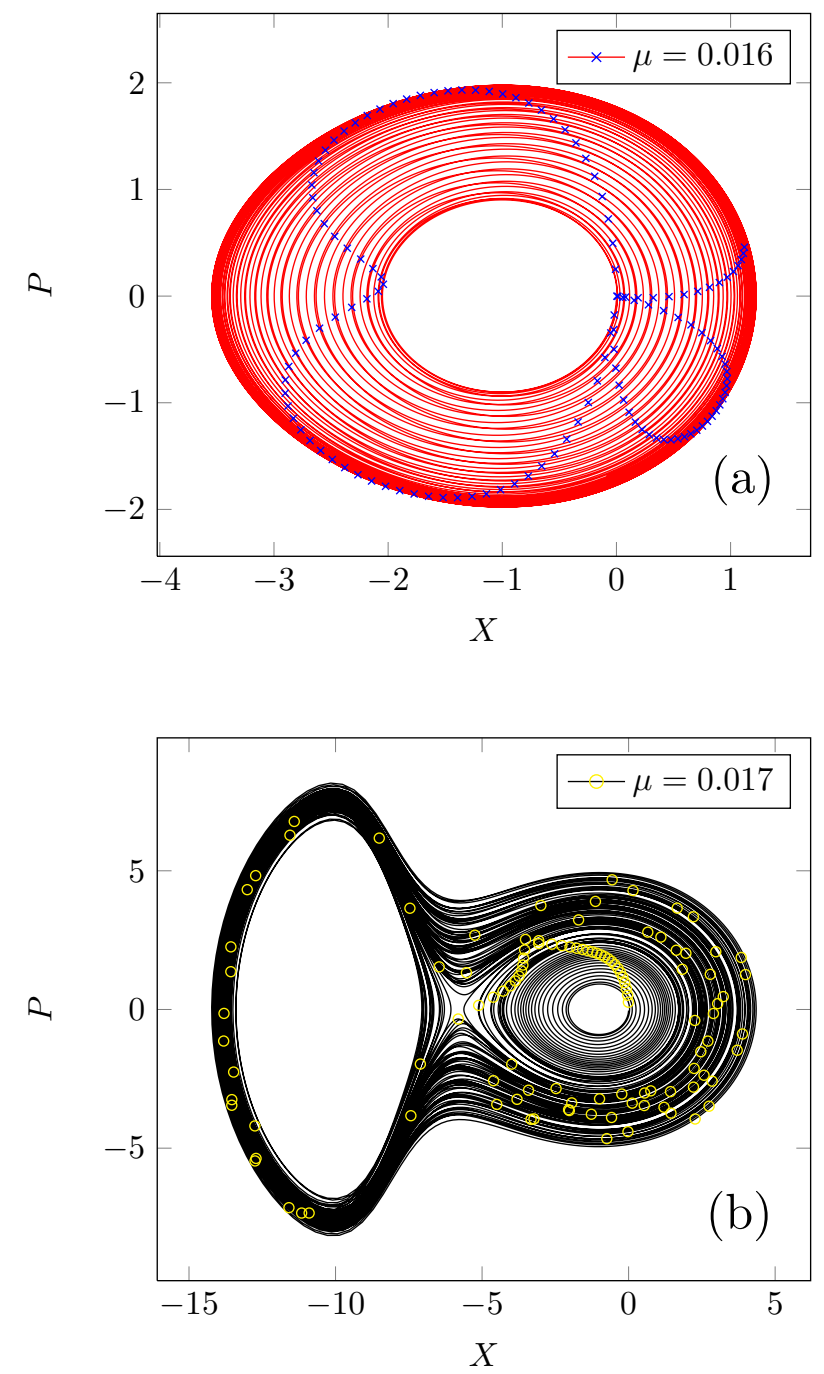

FIG. 7: (Color online.) Phase-space orbits and their associated Poincaré projections for $\eta / \kappa=0.90$, for the case (a) immediately before $(\mu=0.016$, red lines and blue crosses) (b) and after ( $\mu=0.017$, black lines and yellow circles) the modulation-induced crossing of the separatrix, showing quasiperiodic and chaotic behaviour (the yellow circles describe initially quasiperiodic oscillations in the inner part of the Figure, before becoming scattered chaotically across the extended phase-space orbit, respectively.

we demonstrate that when both atom-atom interactions and pump modulation are present simultaneously, some features of the dynamical behaviour of the system are changed with respect to that when only pump modulation or atom-atom interactions are present individually.

We again consider an experimental configuration depicted in Fig. 1. For this configuration and the parameter values listed in section 2, the atom-atom interaction coefficient $\Lambda=157$. Note that this choice of configuration does not affect the generality of our results as very similar behaviour is also observed when the condensate is aligned along the axis of the cavity (which has the effect

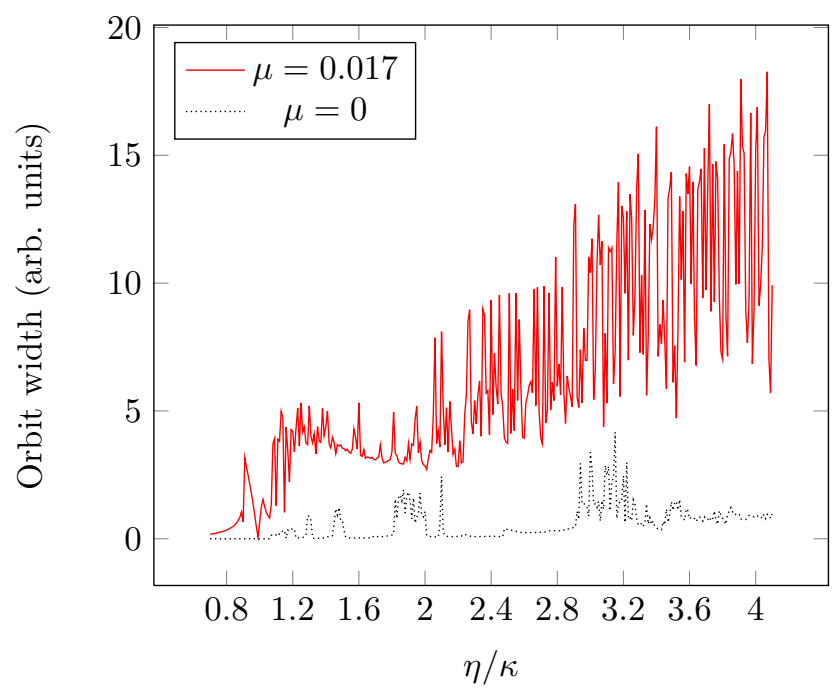

FIG. 8: (Color online.) The width of phase-space orbits for increasing rates of the pump $\eta$, for the case without a pumpbeam modulation ( $\mu=0.00$, black line), and for a small modulation $\mu=0.017$ at frequency $\omega_{m}=0.81 \omega_{r}$ (red line). At all points, the small modulation provokes phase-space excursions whose extent exceeds greatly the no-modulation case. Note that there is additionally an instance of chaos around $\eta=0.90-0.93$ for $\mu=0.017$, which comes before the critical value of $\eta$ for the onset of chaos in the no-modulation case, $\eta_{\mathrm{cr}} \approx 1.07$.

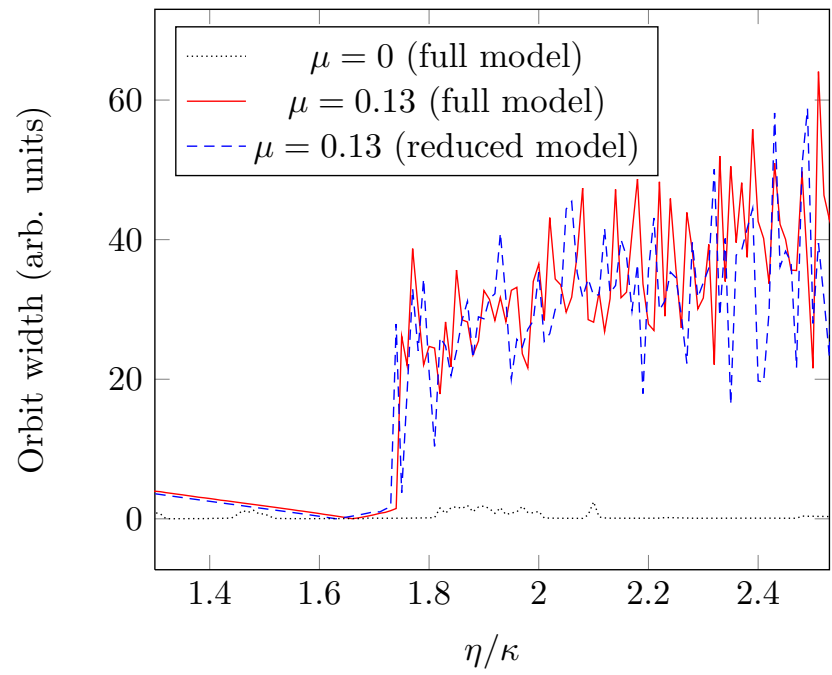

FIG. 9: (Color online.) The width of phase-space orbits for increasing $\eta$, for the case with atom-atom interactions $(\Lambda=$ $157)$ without a pump-beam modulation $(\mu=0.00$, dotted black line), and with modulation $\mu=0.13$ at frequency $1.49 \omega_{r}$ (red line). As in Fig. 8 the modulation has the effect of substantially increasing the extent of phase-space excursions beyond the critical pump rate. Also shown is the orbit width for the case with only five spatial modes considered (dashed blue line); note that it exhibits behaviour similar to that of the full model. 
of increasing $\Lambda$ [19]).

With atomic collisions now included in the model, the BEC-cavity system features two nonlinearities: one arising from the atom-light coupling, and another from atomic collisions. The introduction of this additional nonlinear term alters the effect of the pump modulation, causing a shift of the chaotic resonances to higher modulation frequencies (see Fig. 4). The lowest threshold value of the modulation amplitude $\mu$ required to induce chaos when no atom-atom interactions are present $(\Lambda=0)$ is achieved when $\omega_{m}=0.81 \omega_{r}$. When repulsive atom-atom interactions are included $(\Lambda=157)$, this frequency is shifted to $\omega_{m}=1.49 \omega_{r}$, in agreement with the shift in the unmodulated frequency discussed in Section 3 and in Eq. (7). A further consequence of the inclusion of atomatom interactions $(\Lambda>0)$ is that the values of pump modulation amplitude, $\mu$, required to instigate chaotic dynamics in the system are, in general, greatly increased: for example, at $\omega_{m}=1.49 \omega_{r}$, the threshold value of the pump modulation amplitude, $\mu=0.13$, is much larger than the corresponding value $(\mu=0.017)$ in the case where atom-atom interactions are not present $(\Lambda=0)$.

The inclusion of atom-atom interactions also affects the transition to chaos with increasing pump rate $\eta$. Previous studies [19, 27] have shown that in the absence of pump modulation, the range of $\eta$ over which the BECcavity system exhibits bistability is reduced when repulsive atom-atom interactions are included $(\Lambda>0)$, relative to that when atom-atom interactions are neglected $(\Lambda=0)$. A consequence of this is that the critical pump rate $\eta_{\text {cr }}$ required to induce chaos is larger for a repulsive condensate $(\Lambda>0)$ than for a collisionless condensate $(\Lambda=0)$. This remains the case when pump modulation is also present $(\mu>0)$, as can be seen by a comparison of Fig. 8 and 9 .

Fig. 8 and 9 also show that irrespective of whether atom-atom interactions are present (Fig. 9) or not (Fig. 8), once the pump rate, $\eta$, exceeds a threshold value, $\eta_{\mathrm{cr}}$, the system dynamics become exclusively chaotic. Above this threshold it can be seen that the orbit widths and consequently the phase-space volume and degree of chaos in the modulated pump case $(\mu>0)$ is far greater than in the system without pump modulation $(\mu=0)$. Similarly it can be seen the the degree of chaos in the system dynamics can be maximised when both pump modulation and repulsive atom-atom interactions are present.

\section{REDUCED $n$-MODE MODEL}

Fig. 10 shows that for the BEC-cavity system with modulated input pumps, the number of significant motional states, or spatial modes, of the BEC is very small since we are using a low pump rate to ensure that $\eta<\eta_{\mathrm{cr}}$. Consequently, it is reasonable to model the BEC-cavity system by making use of a simplified model that involves a reduced number of such states. Previous studies (e.g., 21]) have demonstrated that a two-mode model can de-

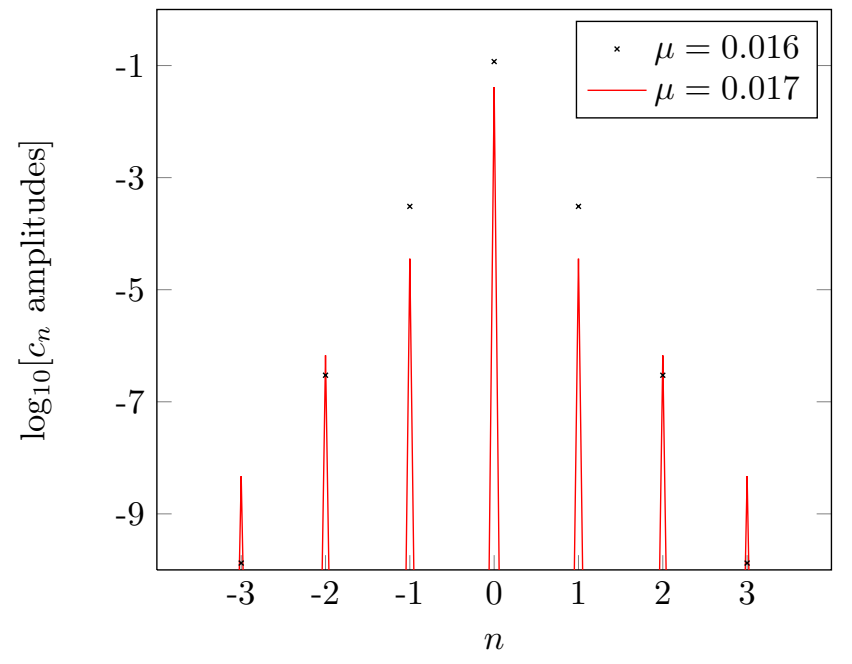

FIG. 10: (Color online.) Spatial BEC spectra averaged over the duration of the BEC-cavity system's evolution, for modulation amplitudes $\mu=0.016$ (black crosses) and $\mu=0.017$ (red lines). The number of relevant modes in both cases is basically the same, and although the $n= \pm 3$ modes are roughly 10 times more prominent for the larger amplitude, their magnitude remains insignificant, suggesting that a fivemode model would be adequate to provide an accurate representation of the system.

scribe a variety of features consistent with the experimental results from the BEC-cavity system. Ref. [19] showed that by increasing the number of spatial modes of the BEC to five, it is possible to describe behaviour not described by the simpler two-mode model, such as the chaotic motion apparent in the evolution of the system described by Eqs. (1) and (2). It is therefore worth investigating whether a model truncated to only five modes can also accurately reflect the system's dynamics when the pump modulation is incorporated.

We proceed by describing the BEC wave function using five spatial modes:

$$
\psi(x, t)=\frac{1}{\sqrt{n_{L}}} \sum_{n=-2}^{2} c_{n}(t) \mathrm{e}^{\mathrm{i} n \pi x},
$$

where $n_{L}=L_{\mathrm{BEC}} /(\lambda / 2)$ is the number of lattice sites occupied by the BEC. Substituting into Eq. (1) then gives

$$
\begin{gathered}
\frac{d c_{n}}{d t}=-\mathrm{i} \frac{n^{2} \pi^{2}}{2} c_{n}-\mathrm{i} \frac{|\alpha|^{2} V_{0}}{2}\left(c_{n}-\frac{c_{n-1}}{2}-\frac{c_{n+1}}{2}\right) \\
-\mathrm{i} \Lambda^{\prime} \sum_{k, m=-2}^{2} c_{k} c_{m}^{*} c_{n+m-k},
\end{gathered}
$$

where $\Lambda^{\prime}=\Lambda / n_{L}$, while the integral in Eq. 22 becomes

$$
\left\langle\psi\left|\sin ^{2}\left(\frac{\pi x}{2}\right)\right| \psi\right\rangle=\frac{1}{2}\left[1-\sum_{n=-2}^{2} c_{n}^{*}\left(c_{n-1}+c_{n+1}\right)\right] .
$$


The results of varying the modulation frequency for the five-mode model, which are shown alongside the results of the full model in Fig. 4, are in good agreement with those of the full model, and accurately reproduce the resonances found at $\omega_{m}=0.81 \omega_{r}$ and $\omega_{m}=1.67 \omega_{r}$ in the full model. Furthermore, the five-mode model is able to accurately predict the onset of chaos in the modulated system, both for the case when the condensate is collision-free $(\Lambda=0)$ and when the atom-atom interactions in the condensate are repulsive $(\Lambda>0)$. An example is the result shown for the five-mode model (dashed blue line) in Fig. 9, compared to that calculated from the full model (red line). It is reasonable to conclude that the essential features of the BEC-cavity system's dynamics are demonstrably captured by the five-mode, low-dimensional model.

\section{CONCLUSION}

It has been shown using numerical simulations that the nonlinear dynamical behaviour of a BEC in an optical cavity is strongly influenced by a periodic modulation of the pump field, and that such a modulation greatly increases the likelihood that the system will display chaotic behaviour. This approach provides a simple mechanism for ensuring chaotic dynamics beyond a critical pump rate, and should be easier to realise in practice than inducing chaos via manipulation of the atom-atom interaction.

It has also been shown that a simplified five-mode model accurately describes the essential features of the BEC-cavity dynamics when the pump is modulated, reproducing the behaviour of the full model for a wide range of parameter values.

The results presented demonstrate that the BECcavity system is rich with possibilities for the investigation of nonlinear dynamics. Possible interesting extensions to the analysis described here include investigations of inhomogeneous initial conditions beyond the assumed spatially uniform state examined here, the possibility of coupled atomic/optical solitonic states, and the viability of exploiting the chaotic regime as the basis of new methods for coherent BEC transport. Extending these investigations from Fabry-Pérot to ring cavities offers the prospect of interesting connections with other dynamical behaviours and instabilities involving light and BECs, e.g., Collective Atomic Recoil Lasing (CARL) 28 30].

An additional extension could be the relaxation of the adiabatic elimination of the cavity field. Until recently, all experiments of the kind described here involved cavities with loss rates such that the timescale of cavity losses $\left(\sim \kappa^{-1}\right)$ was much shorter than the timescale of the BEC dynamics $\left(\sim \Omega_{r}^{-1}\right)$, consistent with adiabatic elimination of the cavity-field dynamics. However, recent experiments 31 33] using ultra high-finesse cavities involve cavity-field dynamics that evolve on a longer timescale than the BEC dynamics, i.e., $\kappa<\Omega_{r}$. How some of the nonlinear phenomena described here will be affected by this new regime is an interesting and open question.

\section{Acknowledgments}

We acknowledge financial support from the Engineering and Physical Sciences Research Council (Grant No. $\mathrm{EP} / \mathrm{HO} 49339)$.
[1] E. Ott, C. Grebogi, and J. Yorke, Phys. Rev. Lett. 64, 1196 (1990).

[2] F. Arecchi, R. Meucci, G. Puccioni, and J. Tredicce, Phys. Rev. Lett. 49, 1217 (1982).

[3] R. Badii, E. Brun, M. Finardi, L. Flepp, R. Holzner, J. Parisi, C. Reyl, and J. Simonet, Rev. Mod. Phys. 66, 1389 (1994).

[4] C. Law and N. Bigelow, Phys. Rev. A 58, 4791 (1998).

[5] P. Horak and H. Ritsch, Phys. Rev. A 63, 023603 (2001).

[6] D. Nagy, G. Szirmai, and P. Domokos, Eur. Phys. J. D 48, $127(2008)$

[7] J. M. Zhang, F. C. Cui, D. L. Zhou, and W. M. Liu, Phys. Rev. A 79, 33401 (2009).

[8] N. Liu, J. Lian, J. Ma, L. Xiao, G. Chen, J.-Q. Liang, and S. Jia, Phys. Rev. A 83, 033601 (2011).

[9] T. Grießer and H. Ritsch, Optics Express 19, 11242 (2011).

[10] S. Slama, S. Bux, G. Krenz, C. Zimmermann, and P. W. Courteille, Phys. Rev. Lett. 98, 53603 (2007).

[11] F. Brennecke, T. Donner, S. Ritter, T. Bourdel, M. Köhl, and T. Esslinger, Nature 450, 268 (2007).

[12] Y. Colombe, T. Steinmetz, G. Dubois, F. Linke,
D. Hunger, and J. Reichel, Nature 450, 272 (2007).

[13] K. Baumann, R. Mottl, F. Brennecke, and T. Esslinger, Phys. Rev. Lett. 107, 140402 (2011).

[14] S. Bux, C. Gnahm, R. A. W. Maier, C. Zimmermann, and P. W. Courteille, Phys. Rev. Lett. 106, 203601 (2011).

[15] V. S. Filho, A. Gammal, T. Frederico, and L. Tomio, Phys. Rev. A 62, 033605 (2000)

[16] P. Coullet and N. Vandenberghe, J. Phys. B: At., Mol. Opt. Phys. 35, 1593 (2002).

[17] J. Kronjger, K. Sengstock, and K. Bongs, New J. Phys. 10, $045028(2008)$

[18] K. Zhang, W. Chen, M. Bhattacharya, and P. Meystre, Phys. Rev. A 81, 013802 (2010).

[19] M. Diver, G. R. M. Robb, and G.-L. Oppo, Phys. Rev. A 89, 033602 (2014).

[20] B. Chen, C. Jiang, and K.-D. Zhu, J. Opt. Soc. Am. B 28, 2007 (2011)

[21] F. Brennecke, S. Ritter, T. Donner, and T. Esslinger, Science 322, 235 (2008).

[22] S. Ritter, F. Brennecke, K. Baumann, T. Donner, C. Guerlin, and T. Esslinger, Appl. Phys. B 95, 213 
(2009).

[23] L. Salasnich, A. Parola, and L. Reatto, Phys. Rev. A 65, 043614 (2002)

[24] P. Louis, E. Ostrovskaya, C. Savage, and Y. Kivshar, Phys. Rev. A 67, 013602 (2003).

[25] N. K. Efremidis and D. N. Christodoulides, Phys. Rev. A 67, 063608 (2003).

[26] R. Carretero-González, D. J. Frantzeskakis, and P. G. Kevrekidis, Nonlinearity 21, 139 (2008).

[27] D. Nagy, G. Szirmai, and P. Domokos, Eur. Phys. Jour. D 67, 124 (2013).

[28] N. Piovella, M. Gatelli, and R. Bonifacio, Opt. Comm. 194, 167 (2001).
[29] R. Bonifacio, M. M. Cola, N. Piovella, and G. R. M. Robb, Europhys. Lett. 69, 55 (2005)

[30] L. Fallani, C. Fort, N. Piovella, M. Cola, F. S. Cataliotti, M. Inguscio, and R. Bonifacio, Phys. Rev. A 71, 033612 (2005)

[31] M. Wolke, J. Klinner, H. Keßler, and A. Hemmerich, Science 337, 75 (2012).

[32] H. Keßler, J. Klinder, M. Wolke, and A. Hemmerich, New J. Phys. 16, 053008 (2014).

[33] H. Keßler, J. Klinder, M. Wolke, and A. Hemmerich, Phys. Rev. Lett. 113, 070404 (2014). 